\title{
Laser induced phase transition in iron thin films
}

\author{
V.S. TEODORESCU, I.N. MIHǍILESCU, M. DINESCU, N. CHITICÃ, L.C. NISTOR*, \\ J. VAN LANDUYT* ${ }^{*}$ and A. BARBORICA** \\ Institute of Atomic Physics, IFTM, IFTAR, P.O. Box MG-6, 76900 Bucharest, Romania \\ * University of Antwerpen, RUCA -EMAT, Groenenborgerlaan 171, 2020 Antwerp, Belgium \\ ** University of Bucharest, Physics Department, P.O. Box Mg-11, 76900 Bucharest, Romania
}

\section{Introduction}

High power laser pulse irradiation of material surfaces produces a wide spectrum of phenomena, all connected with emerging new technologies. Topics like: ablation, thin films deposition, laser induced chemical reaction are frequently studied. Fow studics have been reported in the domain of laser induced solid state phase transitions at a relative low laser fluence. In this regime the laser pulse acting like a high power heat pulse, the evolution of the system is far from equilibrium and new structures or new mechanism of transformation is expected.

In the case of laser thin films heating, the heating rate is of the order of $10^{10} \mathrm{Ks}^{-1}$. If the laser pulse is absorbed only in the thin film and the film is laser irradiated while on the substrate, the rate of cooling can be equally fast, the cold substrate acting as a thermal sink. The thin film is suddenly heated and rapidly cooled, quenching the structure resulting after the laser pulse heating.

In a previous paper ${ }^{1}$ the microstructural aspects of the b.c.c. f.c.c. phase transition of iron thin films were studied by high resolution transmission electron microscopy. This transition of iron is one of the best known and studied allotropic transformation, mainly in the case of martensitic transformation ${ }^{2}$. In the first paper $^{1}$, it was pointed out a new aspect of the phase transition process in the laser heating case, by revealing the appearance of a microscopic mixture of the b.c.c. and f.c.c. phases. This mixture represents an incomplete transformation in the same crystalline block and can be understood as a frozen-in stage of the transition. In this paper we report numerical analysis of the laser 
heating and the correlation with the observed characteristic microstructure.

\section{Experimental}

Iron thin films have been deposited at $R T$ by evaporation in a vacuum of $2 \times 10-6$ torr using $99.9 \%$ iron needles. Freshly cleaved KCl single crystals have been used as substrate. The thickness of the iron films was $40 \mathrm{~nm}$. To prevent oxidation of the iron film, before and after iron cvaporation, two $5 \mathrm{~nm}$ thick amorphous carbon layers were subsequently deposited while still in vacuum. A sandwich film, C-Fe-C, with $50 \mathrm{~nm}$ total thickness was thus obtained.

KrF excimer laser irradiations $(\lambda=248 \mathrm{~nm})$ have been performed in air. The thin films were irradiated by a single pulse on the $\mathrm{KCl}$ substrate. The laser fluence was in the range of 50 to $100 \mathrm{~mJ} \cdot \mathrm{cm}^{-2}$ and the pulse duration $\tau_{\mathrm{FWHM}}=20 \mathrm{~ns}$. The laser fluence was set in such a way that a minimum modification of the film could be observed by optical microscopy. After irradiation on the $\mathrm{KCl}$ substrate, the film is floated of in water and, the irradiated areas, mounted on TEM grids.

\section{Numerical analysis}

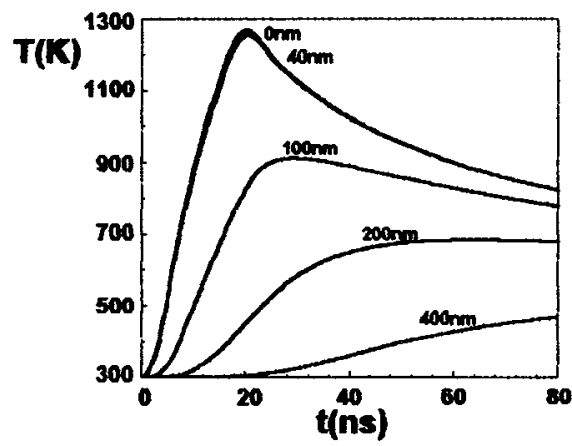

Fig. 1: Temperature evolution of points located at different depths from the surface of the irradiated sample.

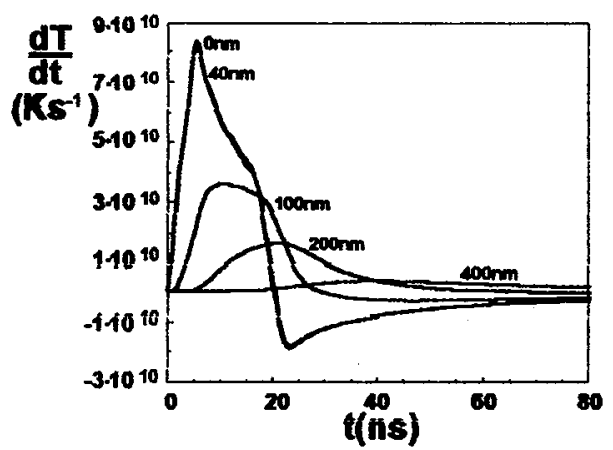

Fig. 2: Evolution of the temperature derivative for points located at different depths.

We made a numerical analysis of the laser heating and subsequent cooling of the irradiated samples using a finite-difference method to 
solve the heat-flow equation ${ }^{3,4}$. In the case of the iron film deposited onto $\mathrm{KCl}$ substrate, we performed a $1-\mathrm{D}$ numerical analysic. This option is based upon the observation that the heat diffusion length $L_{\mathrm{T}}=\sqrt{\chi \tau}(\chi$ is the thermal diffusivity) in the Fe film and in the $\mathrm{KCl}$ substrate on a time cale significant for our analysis $(\tau=100 \mathrm{~ns})$ is about $\nu_{\mathrm{T}} \approx 1 \mu \mathrm{m}(\mathrm{Fe})$ and $L_{\mathrm{T}} \approx 250 \mathrm{~nm}(\mathrm{KCl})$. Therefore, there is no significant lateral heat diffusion as compared to the dimensions of the laser spot, $d \approx 1 \mathrm{~mm}$, and the heat-flow process is significant only over a direction normal to the sample surface. Relevant results of the 1-D numerical analysis are presented in figs. 1 and 2 , presenting the temperature and its time derivative evolution in time for points located at different depths from the surface.

\section{Results and discussions}

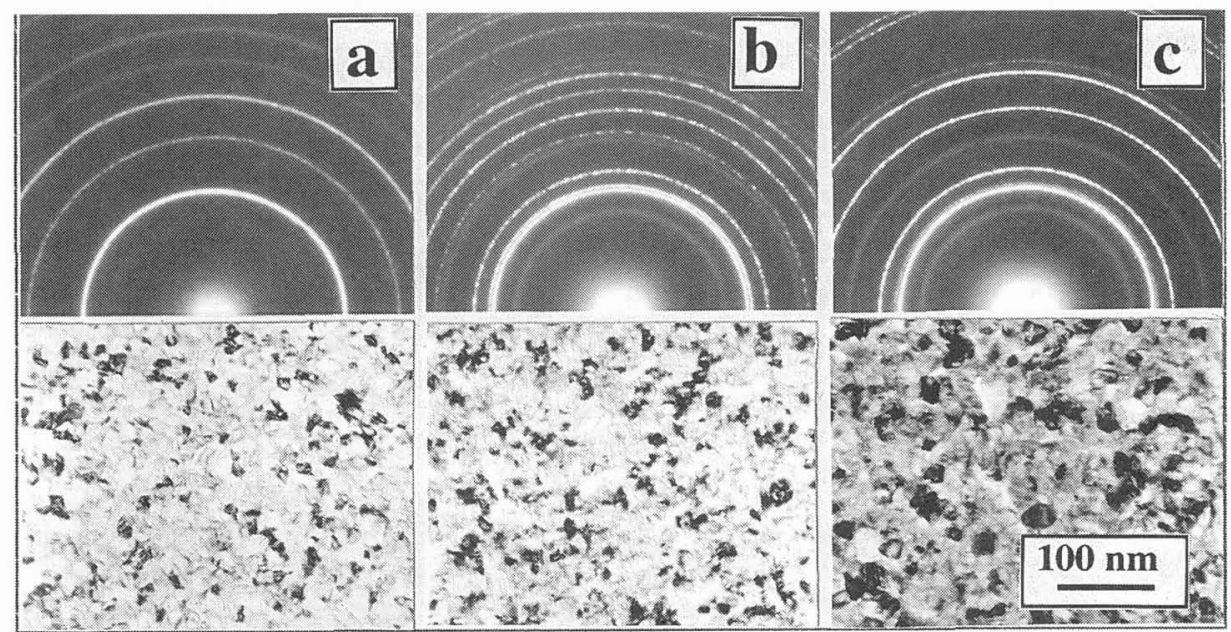

Fig. 3. TEM and corresponding SAED images of the characteristic areas of the C-Fe-C irradiated thin films: (a) b.c.c. phase area, (b) mixture phase area, (c) f.c.c. phase area.

Fig. $3 \mathrm{a}$ illustrate the microstructure of the as deposited thin iron film in the b.c.c. phase with the lattice parameter $a=0.287 \mathrm{~nm}$. In fig. $3 \mathrm{c}$ the f.c.c. microstructure resulting after the phase transition is presented. Between the two phase the irradiated thin film shows a region with a mixed b.c.c. and f.c.c. phase as visible in fig. $3 b$. This mixed regions always appeared between the b.c.c. and f.c.c. areas and have an extension in the film plane of about 10 to $20 \mathrm{~mm}$. High resolution images showed that during the laser heating a crystalline growth 
process takes place. The dimensions of the crystallites grow from about $15 \mathrm{~nm}$ to about $30 \mathrm{~nm}$, mainly by diffusion assisted crystal growth mechanism. . No carbide reflections have been found in the transformed film. The passivating oxide layer which appeared in time on the iron film is not modified by the transformation process.

We ascume that the transition temperature from the b.c.c. to the f.c.c. phase is similar in the thin film case as in the bulk (i.e. 1200$1300 \mathrm{~K}$ ). The numerical calculation in fig. 1 shows that in the thin film the temperature evolution is practically uniform in depth and the temperature peak is quite eymmetrical in time. The detail of the temperature derivative in fig. 2 reveals some differences between the heating and the cooling rate of the film, but the order of magnitude is over $10^{10} \mathrm{Ks}^{-1}$ for both cases.

The driving force for the transformation is proportional to the superheating of the film on the peak of the laser pulse. The superheating in the mixed phase areas of the irradiated films is low and with only 1 or $2 \mathrm{~ns}$ time duration. In this case an incomplete transformation can be observed and the boundaries of the crystailites are mainly phase boundaries. The transformation takes place during temperature rise period and can be considered a revere martencitic trancformation. The transition is very fast, faster than processes like bulk diffusion or oxidation. The b.c.c. and the f.c.c. relative orientation most frequently present in the mixed phase area of the irradiated films is of the Bain type, which can be a characteristic of the reverse martensitic transformation. The laser pulse heating main characteristic is the ability to separate between the solid state trancformation and the diffusive mechanism of structural or chemical transformation.

\section{References}

I V.S. Teodorescu, L.C. Nistor, J. Van Landuyt, Maria Dinescu, accepted at Materiale Research Bulletin (1993).

2 Z. Nishiyama, Martensitic Transformation, Academic Press, N.Y., San Francisco, London (1978).

3 A. Barborică, N. Chiţică, Maria Dinescu, A. Petriø̧, I.N. Mihăilescu, I. Ursu, Phys. Stat. Solidi, 139, 119 (1993).

4W.M. Steen, Laser Materials Processing, Springer-Verlag, London, Berlin, 1991. 